\title{
Acoustic Clicks Activate both the Canal and Otolith Vestibulo-Ocular Reflex Pathways in Behaving Monkeys
}

\author{
Youguo Xu, ${ }^{1}$ Ivra Simpson, ${ }^{1}$ Xuehui Tang, ${ }^{1}$ and Wu Zhou ${ }^{1,2,3}$ \\ ${ }^{1}$ Department of Otolaryngology and Communicative Sciences, University of Mississippi Medical Center, 2500 North State Street, \\ Jackson, MS 39216, USA \\ ${ }^{2}$ Department of Neurology, University of Mississippi Medical Center, Jackson, MS 39216, USA \\ ${ }^{3}$ Department of Anatomy, University of Mississippi Medical Center, Jackson, MS 39216, USA
}

Received: 6 August 2008; Accepted: 16 June 2009; Online publication: 21 July 2009

\begin{abstract}
Acoustic activation of the vestibular system has been well documented in humans and animal models. In the past decade, sound-evoked myogenic potentials in the sternocleidomastoid muscle (cVEMP) and the extraocular muscles (oVEMP) have been extensively studied, and their potentials as new tests for vestibular function have been widely recognized. However, the extent to which sound activates the otolith and canal pathways remains controversial. In the present study, we examined this issue in a recently developed nonhuman primate model of acoustic activation of the vestibular system, i.e., sound-evoked vestibuloocular reflexes (VOR) in behaving monkeys. To determine whether the canal and otolith VOR pathways are activated by sound, we analyzed abducens neurons' responses to clicks that were delivered into either ear. The main finding was that clicks evoked short-latency excitatory responses in abducens neurons on both sides. The latencies of the two responses, however, were different. The mean latency of the contralateral and ipsilateral abducens neurons was $2.44 \pm 0.4$ and $1.65 \pm 0.28 \mathrm{~ms}$, respectively. A further analysis of the excitatory latencies, in combination with the known canal and otolith VOR pathways, suggests that the excitatory responses of the contralateral abducens neurons were mediated by the contralateral disynaptic VOR pathways that connect the lateral canal to the contralateral abducens neurons, and the excitatory responses of the ipsilateral abducens neurons were mediated by the ipsilateral monosynaptic VOR pathways that connect the utricle to the ipsilateral abducens neurons. These results
\end{abstract}

Correspondence to: Wu Zhou $\cdot$ Department of Anatomy $\cdot$ University of Mississippi Medical Center · Jackson, MS 39216, USA. Telephone: +1-601-9845055; fax: +1-601-9841655; email: wzhou@ent.umsmed. edu provide new insights into the understanding of the neural basis for sound-evoked vestibular responses, which is essential for developing new tests for both canal and otolith functions in humans.

Keywords: VEMP, sound activation of vestibular system, Tullio phenomenon

\section{INTRODUCTION}

The vestibular system naturally responds to head accelerations in space. However, it can also be activated by sound because the vestibular end organs and the hearing end organs are connected by continuous fluid in the same membranous labyrinth. Sound activation of the vestibular system was first demonstrated by Tullio in 1929 in pigeons with fenestrated semicircular canals. Over the past several decades, it has been confirmed in other animal models with intact labyrinths (Young et al. 1977, in squirrel monkeys; McCue and Guinan 1994, in cats; Murofushi et al. 1995, in guinea pig; Carey et al. 2004, in chinchilla). In 1994, Colebatch et al. further identified a sound-evoked vestibular response in humans by recording click-triggered averaged EMG from the contracted sternocleidomastoid muscles. This response has been referred to as the vestibular evoked myogenic potential or the VEMP. Because the VEMP offers simplicity, selectivity, and the capacity to stimulate each labyrinth separately, it has been recognized as a promising new test of vestibular function.

Although the vestibular origin of the VEMP has been well established in the original study of Colebatch et al. (1994), the relative contributions of the semicircular canals and the otoliths were not 
investigated until Halmagyi and Colebatch (1995) measured the VEMP in a group of patients with semicircular canal paresis indicated by the absence of caloric irrigation-induced nystagmus. Because some of these canal-deficit patients exhibited normal VEMP, they suggested that the semicircular canals did not contribute to the generation of the VEMP. Based on the proximity of the saccule to the footplate of the stapes, Halmagyi and Colebatch (1995) further proposed that the VEMP is generated by acoustic activation of the sacculo-spinal reflex pathways. Over the past decade, numerous studies in humans and animal models have been interpreted to support this saccular hypothesis and the VEMP has been generally used as a test for the saccular function (for review, Halmagyi et al. 2005). It is likely that the saccule contributes to the generation of the VEMP, but the contribution of the semicircular canals cannot be ruled out. For example, given the well-known limitation of the caloric irrigation test, the absence of caloric irrigation evoked nystagmus does not necessarily guarantee the absence of canal function. The canal response to high-frequency stimulation may be preserved and may have contributed to the VEMP reported in the study of Halmagyi and Colebatch (1995). Furthermore, the studies of Young et al. (1977) in squirrel monkey and Carey et al. (2004) in chinchilla showed that sound stimulation activated vestibular afferents innervating either canals or otoliths. Thus, new investigations are needed to reassess sound activation of canal afferents and their contribution to sound-evoked vestibular responses.

We recently showed that acoustic stimulation evoked well-defined eye movements in awake and behaving monkeys (Zhou et al. 2004, 2005, 2007). The goal of the present study was to take advantage of this new model to investigate sound activation of vestibular end organs by analyzing the sound-evoked responses of abducens neurons in behaving monkeys. According to sensory stimulus, two vestibulo-ocular reflexes (VORs) are distinguished. One is the angular VOR (AVOR) that is activated by angular accelerations detected by the semicircular canals. The other one is the linear VOR (LVOR) that is activated by linear accelerations detected by the otolith organs. For the horizontal AVOR, extensive studies in monkeys and cats have established that the direct AVOR pathways are mainly organized in a disynaptic threeneuron-arc pattern, i.e., from the lateral canal afferents to the interneurons in the vestibular nuclei, then to the abducens motoneurons that innervate the lateral rectus muscle of the contralateral eye (Lorente De No, 1933; Scudder and Fuchs 1992). For the horizontal LVOR, Uchino et al $(1994,1996)$ showed that utricule afferents make monosynaptic excitatory connections to the abducens motoneurons that innervate the lateral rectus muscle of the ipsilateral eye in cats. Thus, by analyzing the sound-evoked responses in abducens neurons on both sides, we were able to determine how clicks activate the canal and otolith VOR pathways in behaving monkeys.

\section{MATERIAL AND METHODS}

Experiments and surgical procedures were performed in accordance with the National Institutes of Health Guide for the Care and Use of Laboratory Animals and were approved by the University of Mississippi Medical Center's Institutional Animal Care and Use Committee.

Eye movement and single-unit recordings

Two Macaca mulatta monkeys (Monkey W and Monkey R) were prepared for chronic recording of binocular eye movements and single-unit recordings in the abducens nucleus. Search coils were surgically implanted in both eyes to record binocular eye movements with a spatial resolution of $\sim 0.01^{\circ}$ (Robinson 1963). Standard procedures were used to record single-unit activity (for more details, see Zhou and King 1998; Zhou et al. 2001). Briefly, a stainless steel cylinder was implanted stereotaxically, and a tungsten microelectrode was advanced through a 21gauge guide cannula by a motorized microdrive. The abducens nucleus was identified by the characteristic tonal quality of the background activity as heard on the audio monitor (Fuchs et al. 1988; Robinson 1970). We included in our sample only the neurons that were recorded concurrently with the characteristic background activity of the abducens nucleus. For each abducens neuron, we first recorded the neuron's responses during pursuit of a sinusoidal target motion at $0.3 \mathrm{~Hz}, \pm 10^{\circ}$, and fixation of the target in $5^{\circ}$ steps from $-20^{\circ}$ to $20^{\circ}$. We then recorded each abducens neuron's responses to acoustic clicks delivered to each ear. Each acoustic click (1 ms, rarefaction, $130 \mathrm{~dB}$ SPL) was generated by a MA3 microphone amplifier (Tucker-Davis Technologies, Alachua, FL). It was triggered by our behavioral control program and was delivered to a monkey's external ear canal via an insert earphone (ER-3A). By measuring the output of a sound level meter (Brüel and Kjoer, Type1613, Copenhagen, Denmark), the onset of the rarefaction near the tympanic membrane was accurately measured to determine the latency of abducens neurons' responses to clicks. Since the insert earphone reduced the bone conductance-induced stimulation of the contralateral labyrinth by $>75 \mathrm{~dB}$, the stimulation of the contralateral labyrinth by clicks used in this study was $<55 \mathrm{~dB}$ SPL, which was lower than the threshold of $80 \mathrm{~dB}$ SPL at which clicks can evoke observable 
behavioral and neuronal responses. Thus, the clicks used in this study provided unilateral vestibular stimulation.

\section{Behavioral paradigms}

During experiments, the monkey was comfortably seated in a custom fabricated chair. Its head was in upright position and stabilized with respect to the electromagnetic field of the eye coil system by attaching a stainless steel rod to the monkey's head holder. Each eye coil was calibrated at the start of each experimental day by requiring the monkey to fixate on known target positions $\left( \pm 20^{\circ}\right.$, every $\left.10^{\circ}\right)$. The positive eye position values were referred to right or up gaze and negative eye position values were referred to left or down gaze.

Monkeys were trained to fixate on visual targets for apple juice rewards. Visual targets were projected by lasers onto a far screen located $\sim 275 \mathrm{~cm}$ from monkeys' eyes. Acoustic clicks were delivered in individual trials that lasted about $4 \mathrm{~s}$. Each trial started with the appearance of a visual target of randomly chosen ocular eccentricity $\left(-20^{\circ},-10^{\circ}, 0^{\circ}, 10^{\circ}, 20^{\circ}\right)$. The monkey was trained to fixate on the target with both eyes, i.e., maintain eye position within a small window $\left(1-4^{\circ}\right.$ in size) centered on the target's position. After a successful fixation interval varying from 300 to $900 \mathrm{~ms}$, a click was delivered to one of the monkey's ears every $400 \mathrm{~ms}$ for six times. The monkey was trained to maintain fixation through the trial. At the end of each successful trial, the monkey was rewarded with two drops of juice. To study the effect of eccentricity on the click-evoked responses, trials with differing eccentricities were combined randomly and delivered in one single block. Each condition consisted of approximately 300 stimulations.

\section{Data acquisition and analysis}

A master PC running specialized software controlled the experiments and a CED Power 1401 system (Cambridge Electronics Devices, Cambridge, UK) for data acquisition. Experimental conditions were formulated as temporal sequences of instructions to operate devices (start or stop data acquisition, move target position, turn on and off targets, trigger a sound stimulus, etc.). Single-unit responses were amplified and filtered $(100-10,000 \mathrm{~Hz})$ and a 2-stage, time-amplitude window discriminator was used to discriminate single spikes by detecting its two peaks (Bak Electronics). The acceptance pulse for action potential that recorded the timing of the second peak was written into a CED event channel with a temporal resolution of $0.01 \mathrm{~ms}$. The amplified extracellular voltage trace was sampled at $20 \mathrm{kHz}$ with 16 -bit resolution to allow us accurately measure the waveform of abducens neurons' action potentials so that the latency from click onset to the foot of action potential was determined. Signals of eye movement, target position were sampled at $2 \mathrm{kHz}$ with 16-bit resolution. These signals were stored on a hard disk for offline analyses.

Eye movement responses were analyzed using Spike2 (Cambridge Electronics Devices, Cambridge, UK). Raw eye position data were filtered and differentiated with a band-pass of DC to $100 \mathrm{~Hz}$ to obtain eye velocity data. Trials in which monkeys made a saccade within $50 \mathrm{~ms}$ of the onset of the sound stimuli were rejected (approximately $1 / 3$ rejected). Trials in the data stream were sorted, aligned on the click onset, and averaged ( $\sim 200$ trials per condition) to obtain low-noise estimates of eye velocity as a function of time.

Single-unit responses to acoustic clicks were analyzed using Spike2 and SigmaPlot. To measure the latency of click-evoked neuronal responses, we computed the cumulative probability of evoking a spike for each neuron (Broussard and Lisberger 1992; Broussard et al. 1995). We first measured the latency between the click and the foot of the first action potential for 150 stimuli. We then arranged the latencies in ascending order and paired them with an ascending series of probabilities ranging in equal increments from $1 / 150$ to 1.0 . To estimate the time course of the probability of firing after clicks, we plotted probability of firing as a function of time (Fig. 1C, stim). To take into account the probability of spontaneous firing, we performed the same analysis on each neuron beginning $30 \mathrm{~ms}$ before the click (Fig. 1C, no stim). Linear regression was used to fit a line to the "no stim" data, and the $Y$ value of the line was subtracted from each "stim" value to yield the probability of evoking a spike as a function of time (Fig. 1D). Latency of the click-evoked response was defined as the onset of the abrupt increase in firing probability (Fig. 1D, arrows). This analysis of latency yields much better temporal resolution because the precise latency of each spike is preserved.

\section{RESULTS}

Click-evoked contralateral response was studied in 107 abducens neurons in two monkeys. Among the 107 neurons, the ipsilateral response was studied in 44 neurons. All the contralateral responses were excitatory. The ipsilateral responses, however, were excitatory in 40 neurons and inhibitory in the other four neurons.

Figure 1 shows the responses of a right abducens neuron to clicks that were delivered into the right ear (ipsilateral response, Fig. 1A) and the left ear (contralateral response, Fig. 1B), while the monkey maintained fixation on a target located at right $10^{\circ}$. 
A
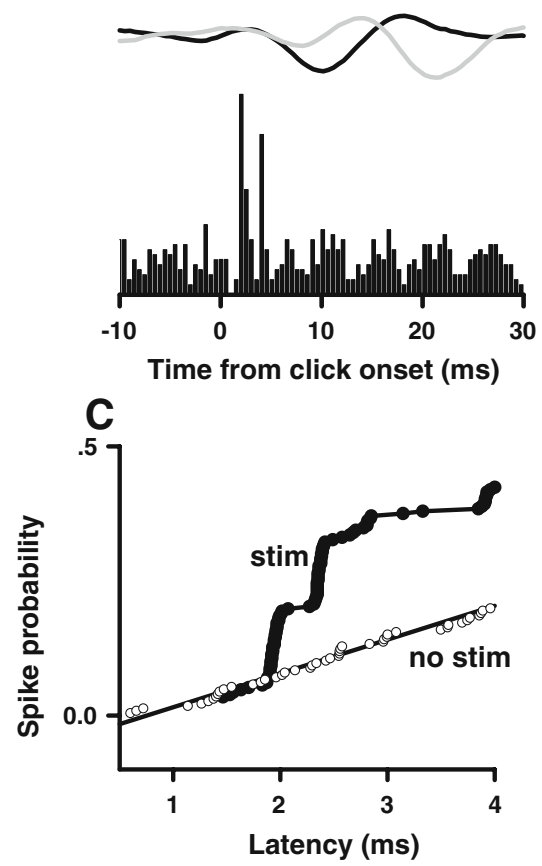

FIG. 1. Click-evoked responses of a typical right abducens neuron (Monkey W). A Right ear click-evoked leftward eye movements and an excitatory response in the neuron. B Left ear click-evoked rightward eye movements and an excitatory response in the neuron. Binned histograms were generated by counting the action potentials within each $0.5 \mathrm{~ms}$ bin from $20 \mathrm{~ms}$ before to $30 \mathrm{~ms}$ after stimulation. The calibration bar is 500 spike/s and $3 \mathrm{deg} / \mathrm{s}$ for firing rate (lower panel) and eye velocity (upper panel), respectively. Black line is for

Excitatory responses were evoked in this neuron when clicks were delivered into either ear. The latency of the click-evoked excitatory response was measured by computing the cumulative probability of evoking a spike (Fig. 1C, D). The latency of the ipsilateral excitatory response was $1.87 \mathrm{~ms}$ (Fig. 1D, black arrow). The latency of the contralateral excitatory response was $2.92 \mathrm{~ms}$ (Fig. 1D, gray arrow). Although clicks evoked excitatory responses in abducens neurons on both sides, the click-evoked binocular horizontal eye movements were not always in the abducting direction. For example, when clicks were delivered into the right ear (Fig. 1A), the left eye (gray line) moved in the abducting direction, but the right eye (black line) moved in the adducting direction.

Latencies of both ipsilateral and contralateral excitatory responses were studied in 33 abducens neurons (Fig. 2A). In 32 neurons, the ipsilateral latencies were shorter than the contralateral latencies, with a difference ranging from 0.03 to $1.09 \mathrm{~ms}$ and a median of $0.87 \mathrm{~ms}$ (ipsilateral $=1.73 \pm 0.24 \mathrm{~ms}$; contralateral $=2.49 \pm 0.3 \mathrm{~ms}, N=33$; paired $t$ test, $p<0.001)$.
B

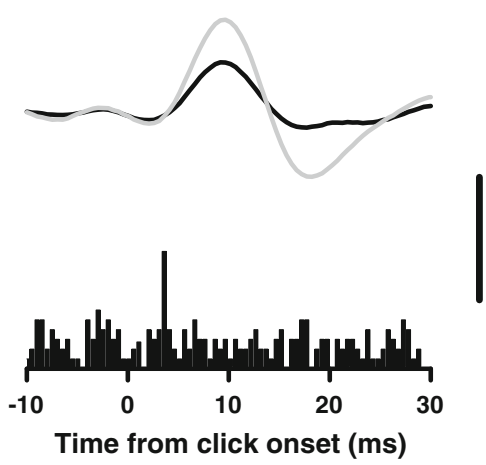

D

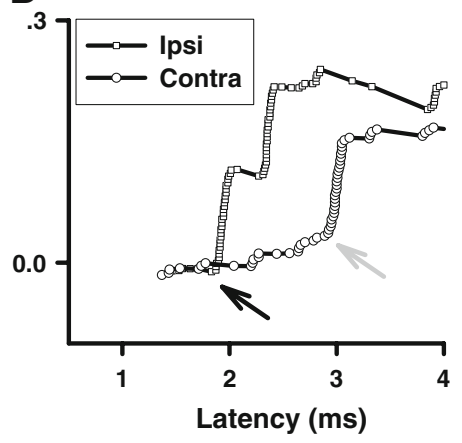

the right eye and gray line for the left eye. C Estimation of the latency of the click-evoked response by computing the cumulative probability of evoking a spike after the click. D Comparison of the latencies of the abducens neuron to ipsilateral (open square) and contralateral (open circle) clicks. The latency to ipsilateral click was $1.87 \mathrm{~ms}$ (indicated by the black arrow). The latency to contralateral click was $2.92 \mathrm{~ms}$ (indicated by the gray arrow).

Similar results were also obtained for the whole population (Fig. 2B, ipsilateral $=1.65 \pm 0.28 \mathrm{~ms}, N=40$; contralateral $=2.44 \pm 0.4 \mathrm{~ms}, N=107, t$ test, $p<0.001$ ). The summed responses of the population were shown in Figure 3 (A for contralateral clicks and B for ipsilateral clicks). Peak response latency was $1.82 \mathrm{~ms}$ for ipsilateral clicks and $2.82 \mathrm{~ms}$ for contralateral clicks.

The click-evoked binocular eye movements were measured at five horizontal gaze eccentricities $\left(-20^{\circ}\right.$, $\left.-10^{\circ}, 0^{\circ},+10^{\circ}, 20^{\circ}\right)$ in two monkeys. Among a total of 40 conditions tested (two monkeys by two ears by two eyes by five gazes), the direction of click-evoked horizontal eye movement was contralateral in 39 conditions (Fig. 4A and the right panel of Fig. 4B). Consistent with an early study (Zhou et al. 2004), their amplitudes decreased as gaze moved toward the stimulated ear. The left panel of Figure 4B, however, reveals a novel feature that was not in the earlier report (Zhou et al. 2004). In this condition (Monkey W, right ear click, left eye, $+20^{\circ}$ ), the direction of click-evoked horizontal eye movement was ipsilateral to the stimulated ear (the thickened line indicated by an arrow). 
A

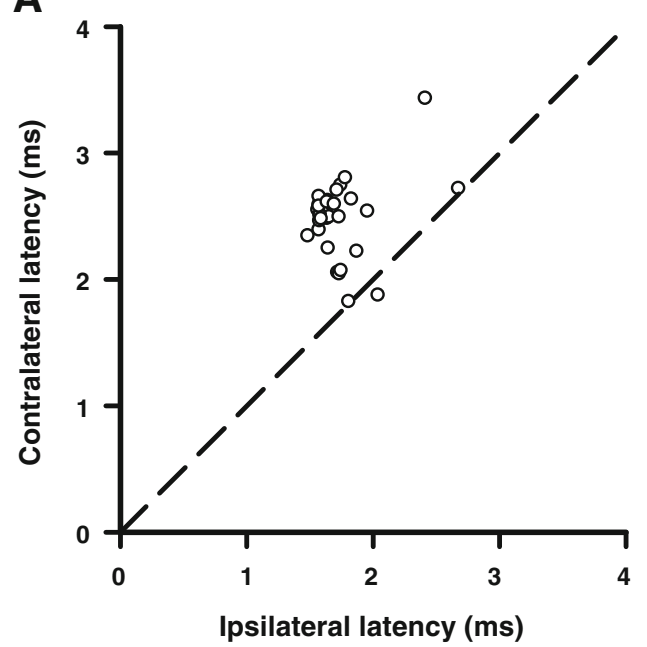

B
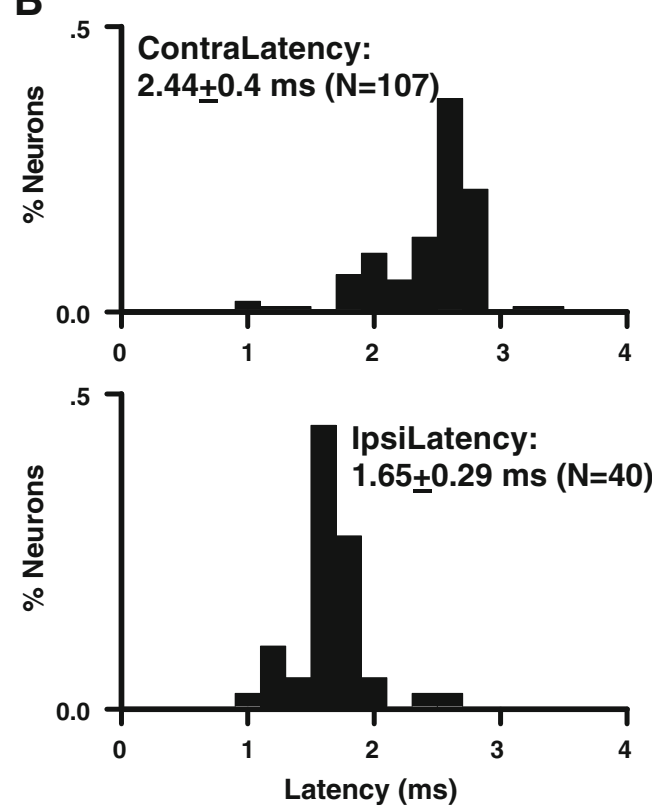

FIG. 2. Comparison of abducens neurons' latencies to ipsilateral and contralateral clicks. A Abducens neurons' latencies to contralateral clicks are plotted against their latencies to ipsilateral clicks. Dotted line is the unitary line. B Distributions of abducens neurons' latencies to ipsilateral clicks (lower panel) and contralateral clicks (upper panel).

\section{DISCUSSION}

\section{Click activates both canal and otolith VOR} pathways

The main finding of the present study was that clicks evoked excitatory responses in abducens neurons on both sides. To elucidate the neural pathways that mediate the two excitatory responses, we measured abducens neurons' latencies to ipsilateral and contralateral clicks and compared them to the published latencies of vestibular afferents and abducens neurons to clicks as well as to brief electrical pulses. As shown in
Figure 5, it has been well established that the contralateral canal-abducens connection is excitatory and disynaptic (Scudder and Fuchs 1992) and the ipsilateral utricular-abducens connection is excitatory and monosynaptic (Uchino et al. 1994, 1996). The latency analysis, in combination with the well-established VOR circuits, provided important insights into understanding how clicks activate specific VOR pathways.

First, we compared the minimum latency of vestibular afferent activation by clicks to that by electrical pulses. Since the two responses have not been studied in the same animal model, data obtained from different animal models were used. This may be justified by the conservative nature of the vestibular peripheral end organs. According to Murofushi et al. (1995), the minimum latency of clickevoked vestibular afferent response was $0.4 \mathrm{~ms}$ in guinea pigs, which was very close to the $0.33 \mathrm{~ms}$ minimum latency of short electric pulse-evoked vestibular afferent response in squirrel monkeys (Goldberg et al. 1984). The extreme short latency is one of the most prominent features of acoustic activation of the vestibular system, but we know little about how it occurs. For example, we do not know the latency from click onset to the movement of the ampulla or otoconia. We also do not know whether clicks activate vestibular afferents via hair cell mechanisms or direct mechanical stimulation of the nerve terminals. Nevertheless, the short minimum latency provides the basis for analyzing the latency of the vestibular nucleus neurons and abducens motoneurons. Second, we compared the minimum latency of abducens neurons' responses to contralateral clicks and that to contralateral electrical pulses. According to Broussard et al. (1995), the minimum latency of crossed, excitatory response was $1.08 \mathrm{~ms}$ in behaving monkeys. In this study, the minimum latency of crossed, excitatory response was $1.02 \mathrm{~ms}$, which was plausible for a response mediated by a disynaptic circuit given the small difference in the vestibular afferent minimum latency to electric pulses and clicks (0.33 vs. $0.4 \mathrm{~ms})$. Third, as shown by several earlier studies (Uchino et al. 1994, 1996, 1997; Isu et al. 2000), the latency analysis involves not only the minimum latency but also the range and distribution of the latency. According to Broussard et al. (1995), the median latency of crossed, excitatory response was $1.5 \mathrm{~ms}$. Given the $0.9 \mathrm{~ms}$ median latency of click-evoked responses of vestibular afferents (Murofushi et al. 1995), the $2.55 \mathrm{~ms}$ median latency of click-evoked crossed, excitatory response of abducens neurons is consistent with the activation of the disynaptic direct VOR pathways. Fourth, we compared the latencies of abducens neurons and the second order vestibular nuclei neurons to ipsilateral clicks. According to Murofushi et al (1996), the latency of second-order 


\section{A. Contralateral Stimulation}

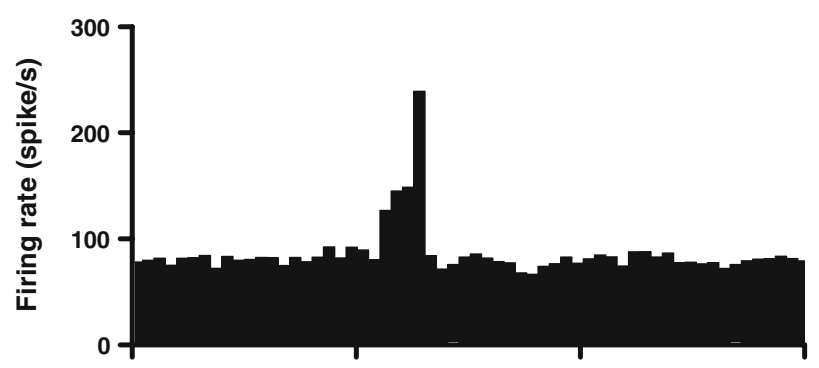

B. Ipsilateral Stimulation

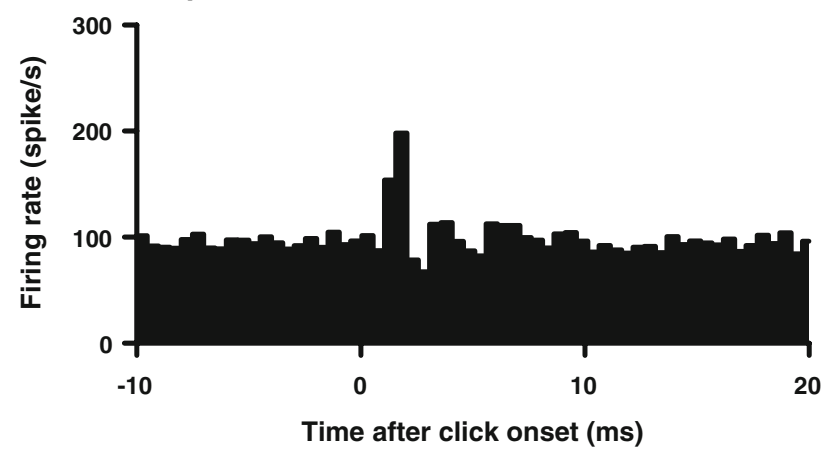

FIG. 3. Composite histograms showing the population's summed responses at the center gaze to contralateral clicks (A) and ipsilateral clicks (B).

vestibular nuclei neurons to ipsilateral clicks was from 1.4 to $3.0 \mathrm{~ms}$ with a mean of $1.75 \mathrm{~ms}$. In this study, we found the abducens latency to ipsilateral clicks ranging from 0.97 to $2.67 \mathrm{~ms}$ with a mean of $1.65 \mathrm{~ms}$ and a median of $1.63 \mathrm{~ms}$. It was consistent with a response that was mediated by a monosynaptic connection. Finally, we compared the latencies of the same abducens neurons to ipsilateral and contralateral clicks. For a group of 33 abducens neurons in which both the ipsilateral and contralateral latencies were measured, the ipsilateral latency was shorter than the contralateral latency in 32 neurons, with a difference ranging from 0.03 to 1.09 ms and a median of $0.87 \mathrm{~ms}$. This difference was consistent with an additional synaptic delay in the circuit mediating the contralateral responses.

In summary, the latency analysis indicates that click-evoked excitatory responses in the contralateral and ipsilateral abducens neurons were mediated by disynaptic and monosynaptic pathways, respectively. To find out the neural pathways that mediate the two excitatory responses, we need to combine the latency analysis with the known horizontal VOR pathways that connect vestibular end organs to the abducens neurons. Although the otolith-ocular pathways are not well understood, accumulating evidence suggests that its organization is very different from that of the canal-ocular pathways (for review, see Angelaki 2004). For example, the canal-ocular pathways are mainly organized within a three-neuron-arc pattern whereby semicircular canal projections through the vestibular nuclei make disynaptic excitatory connections to contralateral abducens motoneurons and inhibitory connections to ipsilateral abducens neurons (Baker et

\section{A. Left ear click}

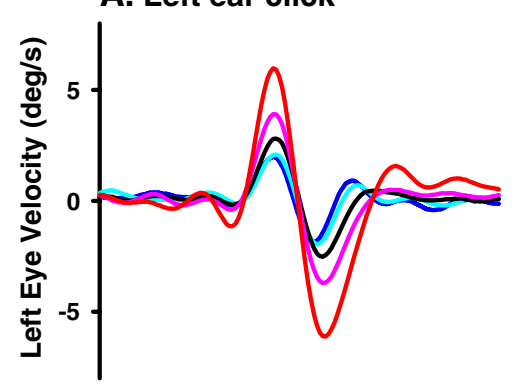

B. Right ear click

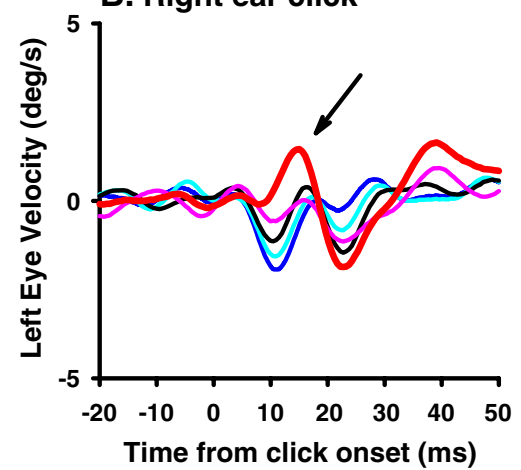

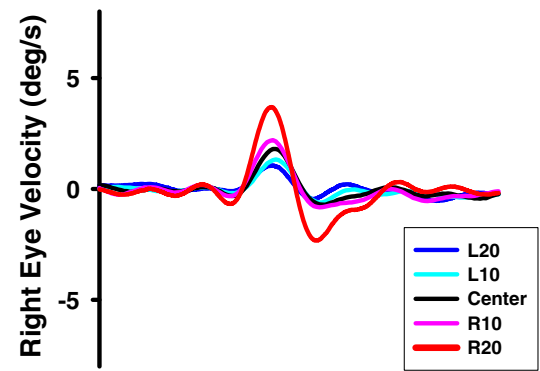

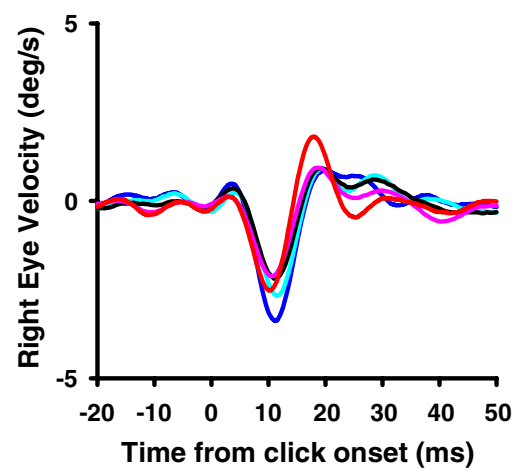

FIG. 4. Click-evoked binocular eye movements as a function of gaze eccentricity (Monkey W). A Clicks were delivered into the left ear. B Clicks were delivered into the right ear. 
A

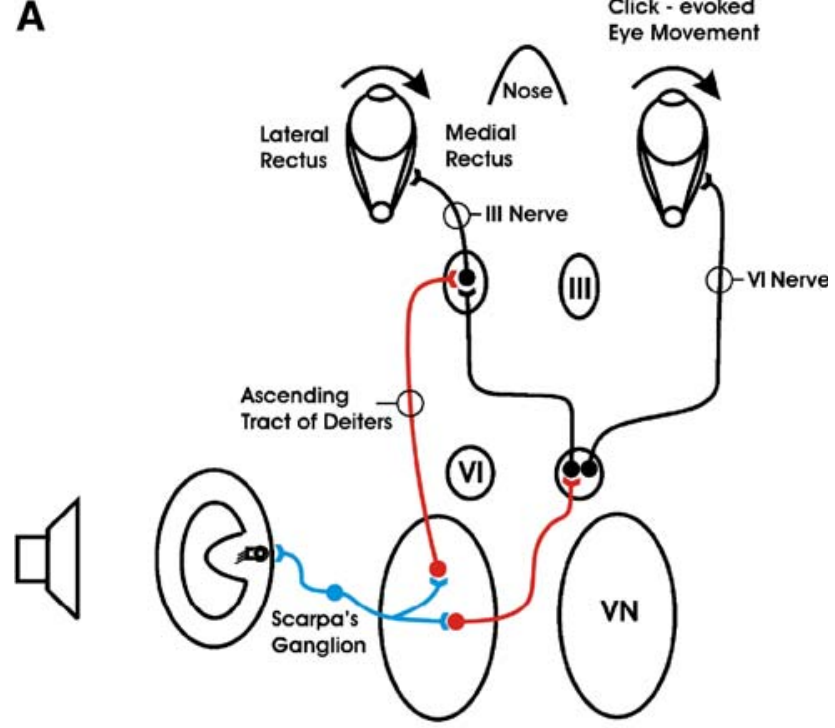

B

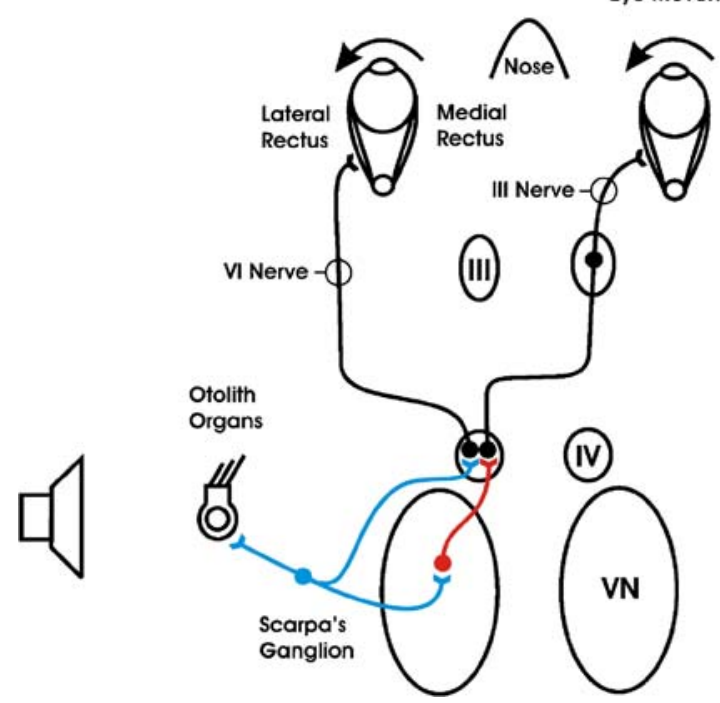

FIG. 5. Separate direct VOR pathways for horizontal canal VOR (A) and utricule VOR (B). Adapted from Angelaki (2004).

al. 1969; Richter and Precht 1968; Schwindt et al. 1973). The otolith-ocular pathways, however, have been shown to have a very different circuitry. Specifically, the utricule projections make monosynaptic and disynaptic excitatory connections to the ipsilateral abducens neurons and trisynaptic inhibitory connections to the contralateral abducens neurons (Imagawa et al. 1995; Schwindt et al. 1973; Uchino et al. 1994, 1996, 1997). The saccular projections make no connections to the horizontal extraocular motoneurons. They only make weak connections to the vertical extraocular motoneurons (Isu et al. 2000). Based on the above-described horizontal VOR circuitry (Fig. 5), the click-evoked monosynaptic ipsilateral excitatory responses of abducens neurons did not originate from the canals but from the utricule (Fig. 5B). The click-evoked disynaptic crossed, excitatory responses, however, did not originate from the utricule nor the saccule but from the lateral canal (Fig. 5A).

\section{Click-evoked eye movements represent sum of multiple components}

Since clicks activate both canal and otolith end organs, they activate several VOR pathways that include the canal-abducens pathways, the otolithabducens pathways, and the canal-oculomotor pathways via the ascending tract of Deiters (Fig. 5A). In the horizontal direction, activation of the contralateral canal-abducens pathways evokes contralateral eye movements in both eyes; activation of the ipsilateral utricule-abducens pathways evokes ipsilateral eye movements in both eyes. Activation of the ascending tract of Deiters, however, evokes contralateral eye movements only in one eye. The observed click-evoked eye movement represented the sum of all these components.

At the center gaze, clicks always evoked contralateral directed eye movements, indicating that the contralateral components were stronger than the ipsilateral components. As gaze eccentricity shifted toward the stimulated ear, the contralateral components decreased and the ipsilateral components increased. Although the sum of these components decreased, its direction was still contralateral in most conditions (Fig. 4). This result was consistent with earlier reports that examined the gaze eccentricity effect of click-evoked eye movements and neuronal responses (Zhou et al. 2004, 2007). It is worth noting, however, that there is one trace in the left panel of Figure $4 \mathrm{~B}$ (indicated by the arrow) that revealed an exception to this general finding. In this condition (Monkey W, right ear, left eye, right $20^{\circ}$ gaze eccentricity), the direction of the click-evoked eye movement was ipsilateral rather than contralateral. A parsimonious interpretation of this exception is that the click-evoked horizontal eye movement was not generated by activating the contralateral labyrinthabducens pathways alone because that would only generate a contralateral eye movement. The ipsilateral excitatory labyrinth-abducens pathways that generate an ipsilateral eye movement were also involved. Since the effects of the two VOR pathways added linearly, the direction of the observed eye movement was determined by the component with larger amplitude. In 39 out of 40 conditions tested, the contralateral component was larger, and the observed eye movement was contralateral. In the exception shown in Figure 4B, however, the ipsilateral 
component was larger and the observed eye movement became ipsilateral.

Since the click-evoked eye movement represents the sum of multiple components, it has limitations for characterizing the specific sound activation of the canal and otolith VOR pathways. Muscle force measurements that distinguish the activity of individual muscles in an "antagonistic pair" may be needed to further analyze the click-evoked activation of the two VOR pathways (Miller and Robins 1992; Miller et al. 2002). Muscle force measurements also better reflect the high-frequency behavior of motoneurons than eye position measurements, which are low-pass filtered by the viscous orbit $(60 \sim 100 \mathrm{~Hz}$, Bahill et al. 1981). Another limitation of the study was that we were unable to identify whether a recorded abducens neuron was a motoneuron that innervates the lateral rectus muscle or an internuclear neuron that projects to the contralateral oculomotor nucleus (Langer et al. 1986; Zhou and King 1998; Clendaniel and Mays 1994; Gamlin et al. 1989; Fuchs et al. 1988; Sylvestre and Cullen 2002). It was likely that our population consisted of neurons from both groups. Recordings in the abducens and oculomotor nerves will allow us to further characterize the click-evoked responses in the canal and otolith VOR pathways.

\section{Sound-evoked responses in vestibular afferents}

Although this is the first study that recorded the clickevoked neuronal responses in the VOR pathways in behaving monkeys, acoustic activation of vestibular end organs has been investigated in several anesthetized animal models (Young et al. 1977, in squirrel monkeys; McCue and Guinan 1994, in cats; Murofushi et al. 1995, in guinea pig; Carey et al. 2004, in chinchilla). These previous studies revealed that both phase locking and tonic changes in firing rate were evoked in the afferents of all five vestibular end organs. Since the duration of the click employed in this study was only $1 \mathrm{~ms}$, the observed VOR responses were primarily due to click-induced phase locking in the firing of a large number of VOR neurons. A recent study in behaving monkeys further showed that the phase-locking behavior of vestibular afferents was evoked by high frequency natural head rotations ( $\sim 50 \mathrm{~Hz}$; Ramachandran and Lisberger 2006).

\section{Sound-evoked vestibular responses in humans}

Recently, a new type of sound-evoked vestibular potential has been recorded from the oVEMP (Jombik and Bahyl 2005; Todd et al. 2007; Welgampola et al., 2009). These ocular potentials appear to be myogenic since they have been shown to precede the associated sound-evoked eye movements. Since the oVEMP measures the sound-evoked responses in the VOR pathways, it is complementary to the cVEMP that measures the sound-evoked responses in the vestibulospinal reflex pathways. Although the eye movement and single-unit recording results obtained in monkeys may not be directly used to interpret the oVEMP results in humans, the co-activation of the canal and otolith VOR pathways by sound suggests that the saccular hypothesis of the cVEMP should not be simply applied to the oVEMP. Furthermore, our results suggest that future studies are needed to determine whether the sound-evoked activation of canals contributes to generation of the cVEMP.

\section{ACKNOWLEDGMENT}

Supported by grants from NIH (DC08585) to Dr. Wu Zhou; we thank Jiachun Cai for writing the data acquisition program and Jerome Allison for technical assistance.

\section{REFERENCES}

ANGELAKI DE. Eyes on target: what neurons must do for the vestibuloocular reflex during linear motion. J. Neurophysiol. 92 (1):20-35, 2004.

Bahill AT, Allan Brockenbrough A, and Troost BT. Variability and development of a normative data base for saccadic eye movements. Invest. Ophthalmol. Vis. Sci. 21 (1 Pt 1):116-125, 1981.

Baker RG, Mano N and Shimazu H. Postsynaptic potentials in abducens motoneurons induced by vestibular stimulation. Brain Res. 15(2):577-580, 1969.

Broussard DM, DeCharms RC and Lisberger SG. Inputs from the ipsilateral and contralateral vestibular apparatus to behaviorally characterized abducens neurons in rhesus monkeys. J. Neurophysiol. 74:2445-59, 1995.

Broussard DM AND Lisberger SG. Vestibular inputs to brain stem neurons that participate in motor learning in the primate vestibuloocular reflex. J. Neurophysiol. 68:1906-1909, 1992.

Carey JP, Hirvonen TP, Hullar TE and Minor LB. Acoustic responses of vestibular afferents in a model of superior canal dehiscence. Otol Neurotol 25:345-352, 2004.

Clendaniel RA AND Mays LE. Characteristics of antidromically identified oculomotor internuclear neurons during vergence and versional eye movements. J. Neurophysiol. 71:1111-1127, 1994.

Colebatch JG, Halmagy GM, Skuse NF. Myogenic potentials generated by a click-evoked vestibulocollic reflex. J. Neurol. Neurosurg. Psychiatry 57:190-197, 1994.

Gamlin PD, GNADT JW AND MaYs LE. Abducens internuclear neurons carry an inappropriate signal for ocular convergence. J. Neurophysiol. 62:70-81, 1989.

Fuchs AF, Scudder CA And Kaneko CR. Discharge patterns and recruitment order of identified motoneurons and internuclear neurons in the monkey abducens nucleus. J. Neurophysiol. 60:1874-95, 1988.

Goldberg JM, Smith CE, and Fernandez CA. Relation between discharge regularity and responses to externally applied galvanic currents in vestibular nerve afferents of the squirrel monkey. J. Neurophysiol. 51(6):1236-1256, 1984.

Halmagy GM and Colebatch JG. Vestibular evoked myogenic potentials in the sternomastoid muscle are not of lateral canal origin. Acta. Oto-Laryngologica. 115(520):1-3, 1995. 
Halmagy GM, Curthoys IS, Colebatch JG and Aw ST. Vestibular responses to sound. Ann. N. Y. Acad. Sci. 1039:54-67, 2005.

Imagawa M, Isu N, Sasaki M, Endo K, Ikegami H and Uchino Y. Axonal projections of utricular afferents to the vestibular nuclei and the abducens nucleus in cats. Neurosci. Lett. 186(23):87-90, 1995.

Isu N, Graf W, Sato H, Kushiro K, Zakir M, Imagawa M and Uchino Y. Sacculo-ocular reflex connectivity in cats. Exp. Brain. Res. 131:262-268, 2000.

Jombik P AND BAHYL V. Short latency responses in the averaged electro-oculogram elicited by vibrational impulse stimuli applied upon the skull: could they reflect the vestibulo-ocular reflex function? J. Neurol. Neurosurg. Psychiatry 76:222-228, 2005.

Langer T, Kaneko CR, Scudder CA and Fuchs AF. Afferents to the abducens nucleus in the monkey and cat. J. Comp. Neurol. 245:379-400, 1986.

Lorente De No R. Vestibulo-ocular reflex arc. Arch. Neurol. Psychiatry 30:245-291, 1933.

McCue MP and Guinan JJ. Acoustically responsive fibers in the vestibular nerve of the cat. J. Neurosci. 14:6058-6070, 1994.

Miller JM AND Robins D. Extraocular muscle forces in alert monkey. Vision Res. 32: 1099-1113, 1992.

Miller JM, Christopher JB and Dmitri SP. Missing lateral rectus force and absence of medial rectus co-contraction in ocular convergence. J. Neurophysiol. 87:2421-2433, 2002.

Murofushi T, Curthoys IS, Topple AN, Colebatch JG and Halmagy GM. Response of guinea pig primary vestibular neurons to clicks. Exp. Brain Res. 103:174-178, 1995.

Murofushi T, Curthoys IS, Gilchrist DP. Response of guinea pig primary vestibular nucleus neurons to clicks. Exp. Brain Res. 111:149-152, 1996

RAMACHANDRAN R AND Lisberger SG. Transformation of vestibular signals into motor commands in the vestibuloocular reflex pathways of monkeys. J. Neurophysiol. 96:1061-1074, 2006.

Richter A ANd Precht W. Inhibition of abducens motoneurons by vestibular nerve stimulation. Brain Res. 11(3):701-705, 1968.

RoBINSON DA. A method of measuring eye movement using a scleral search coil in a magnetic field. IEEE Trans. Biomed. Eng. 10:137-145, 1963.

Robinson DA. Oculomotor unit behavior in the monkey. J. Neurophysiol. 33:393-403, 1970.

Scudder CA AND Fuchs AF. Physiological and behavioral identification of vestibular nucleus neurons mediating the horizontal vestibuloocular reflex in trained rhesus monkeys. J. Neurophysiol. 68:244-264, 1992.

Schwindt PC, Richter A And Precht W. Short latency utricular and canal input to ipsilateral abducens motoneurons. Brain Res. 60(1):259-262, 1973.

Sylvestre PA And Cullen KE. Dynamics of abducens nucleus neuron discharges during disjunctive saccades. J. Neurophysiol. 88:3452-3468, 2002.

Todd NM, Rosengren SM, Aw ST and Colebatch JG. Ocular vestibular evoked myogenic potentials (OVEMPs) produced by air- and bone-conducted sound. Clin. Neurophys. 118:381-90, 2007.

Uchino Y, Ikegami H, Sasaki M, Endo K, Imagawa M and Isu N. Monosynaptic and disynaptic connections in the utriculo-ocular reflex arc of the cat. J. Neurophysiol. 71:950-958, 1994.

Uchino Y, Sasaki M, Sato H, Imagawa M, Suwa H and Isu N. Utriculoocular reflex arc of the cat. J. Neurophysiol. 76:1896$1903,1996$.

Uchino Y, Sato H, Sasaki M, Imagawa M, Ikegami H, Isu N, and Graf W. Sacculocollic reflex arcs in cats. J. Neurophysiol. 77:30033012, 1997.

Welgampola MS, Americo A. Migliaccio AA, Myrie OA, Minor LB and Carey JP. The human sound-evoked vestibulo-ocular reflex and its electromyographic correlate. Clinic Neurophysiol. 120:158-166, 2009

Young ED, Fernandez C and Goldberg JM. Responses of squirrel monkey vestibular neurons to audio-frequency sound and head vibration. Acta Otolaryngol. 84:352-360, 1977.

Zhou W, TANG BF and King WM. Responses of rostral fastigial neurons to linear acceleration in an alert monkey. Exp. Brain Res. 139:111-5, 2001.

Zhou W and King WM. Premotor commands encode monocular eye movements. Nature 393:692-695, 1998.

Zhou W, Mustain W and Simpson I. Sound-evoked vestibulo-ocular reflexes (VOR) in trained monkeys. Exp. Brain Res. 156:129134, 2004.

Zhou W, Simpson I, Xu Y and Fong A. Activity-dependent modulation: a non-linearity in the unilateral vestibulo-ocular reflex (VOR) pathways. Exp. Brain Res. 163:267-272, 2005.

Zhou W, Xu Y and Simpson I. Multiplicative computation in the vestibulo-ocular reflex (VOR), J. Neurophysiol. 97:2780-2789, 2007. 